\title{
THE X-RAY SPECTRAL PROPERTIES AND VARIABILITY OF LUMINOUS HIGH-REDSHIFT ACTIVE GALACTIC NUCLEI
}

\author{
O. Shemmer, ${ }^{1}$ W. N. Brandt, ${ }^{1}$ C. Vignali, ${ }^{2,3}$ D. P. Schneider, ${ }^{1}$ X. Fan, ${ }^{4}$ \\ G. T. Richards, ${ }^{5}$ and Michael A. Strauss ${ }^{5}$ \\ Received 2005 April 8; accepted 2005 May 20
}

\begin{abstract}
We perform a detailed investigation of moderate- to high-quality X-ray spectra of 10 of the most luminous active galactic nuclei (AGNs) known at $z>4$ (up to $z \sim 6.28$ ). This study includes five new XMM-Newton observations and five archived X-ray observations (four by XMM-Newton and one by Chandra). We find that the X-ray powerlaw photon indices of our sample, composed of eight radio-quiet sources and two that are moderately radio-loud, are not significantly different from those of lower redshift AGNs. The upper limits obtained on intrinsic neutral hydrogen column densities, $N_{\mathrm{H}} \lesssim 10^{22}-10^{23} \mathrm{~cm}^{-2}$, indicate that these AGNs are not significantly absorbed. A joint fit performed on our eight radio-quiet sources, with a total of $\sim 7000$ photons, constrains the mean photon index of $z>4$ radio-quiet AGNs to $\Gamma=1.97_{-0.04}^{+0.06}$, with no detectable intrinsic dispersion from source to source. We also obtain a strong constraint on the mean intrinsic column density, $N_{\mathrm{H}} \lesssim 3 \times 10^{21} \mathrm{~cm}^{-2}$, showing that optically selected radioquiet AGNs at $z>4$ are, on average, not more absorbed than their lower redshift counterparts. All this suggests that the X-ray production mechanism and the central environment in radio-quiet AGNs have not significantly evolved over cosmic time. The mean equivalent width of a putative neutral narrow Fe K $\alpha$ line is constrained to be $\lesssim 190 \mathrm{eV}$, and similarly we place constraints on the mean Compton reflection component $(R \lesssim 1.2)$. None of the AGNs varied on short ( $\sim 1 \mathrm{hr}$ ) timescales, but on longer timescales (months to years) strong variability is observed in five of the sources. In particular, the X-ray flux of the $z=5.41$ radio-quiet AGN SDSS 0231-0728 dropped by a factor of $\sim 4$ over a rest-frame period of 73 days. This is the most extreme X-ray variation observed in a luminous $z>4$ radioquiet AGN.
\end{abstract}

Subject headings: galaxies: active — galaxies: nuclei — quasars: individual (SDSS 0231-0728) — X-rays: galaxies

\section{INTRODUCTION}

Active galactic nuclei (AGNs) at $z>4$ are valuable cosmological probes of the physical environment in the $\sim 1$ Gyr old universe, following the reionization epoch at the end of the "dark ages" (e.g., Fan et al. 2002). Investigations of physical processes in these sources, and in particular their energy production mechanism, are important for understanding the ionizing flux contribution from AGNs in the early universe, black hole $(\mathrm{BH})$ growth, and the evolution of mass accretion in galactic nuclei (see Brandt \& Hasinger 2005 for a recent review). It is not yet clear whether the small-scale physics of active nuclei are sensitive to the strong large-scale environmental changes the universe has experienced since $z \approx 6$.

The penetrating X-ray emission from AGNs is of particular interest, since it allows one to probe the innermost region of the accretion flow, near the central supermassive BH. Until fairly recently, X-ray detections of $z>4$ AGNs were limited to a handful of sources with poor photon statistics, preventing a detailed analysis of their X-ray spectral properties (Kaspi et al. 2000); this was also, in part, a consequence of the relatively small number $(\lesssim 100)$ of such sources discovered by the year

\footnotetext{
${ }^{1}$ Department of Astronomy and Astrophysics, Pennsylvania State University, University Park, PA 16802; ohad@astro.psu.edu.

2 INAF-Osservatorio Astronomico di Bologna, Via Ranzani 1, 40127 Bologna, Italy.

3 Dipartimento di Astronomia, Università degli Studi di Bologna, Via Ranzani 1, 40127 Bologna, Italy.

4 Steward Observatory, University of Arizona, 933 North Cherry Avenue, Tucson, AZ 85721.

5 Princeton University Observatory, Peyton Hall, Princeton, NJ 08544.
}

2000. The past five years have seen a rapid increase in optical discoveries of $z>4$ AGNs, largely as a result of two surveys, the Palomar Digital Sky Survey (DPOSS; Djorgovski et al. 1998 ) and the Sloan Digital Sky Survey (SDSS; York et al. 2000), which have raised the number of such sources to $\sim 600$ (e.g., Schneider et al. 2005). A rapid increase in X-ray detections of $z>4$ AGNs soon followed, with $\sim 90$ such sources to date. ${ }^{6}$ Chandra and XMM-Newton observations of these sources have provided constraints on their mean global X-ray spectral properties, in particular their photon index and intrinsic absorption column density (e.g., Brandt et al. 2004; Vignali et al. 2005). However, many of the X-ray observations are shallow, aimed at just detecting the AGN, and do not allow useful measurements of the spectral properties in individual sources (e.g., Brandt et al. 2002; Vignali et al. 2003a, 2005). Exposure times of several tens of ks with Chandra and XMM-Newton are needed to measure the spectral properties accurately. Such observations are important for investigating the energy production mechanism at this early epoch and testing whether the X-ray spectral properties have evolved over cosmic time.

Recent studies of X-ray spectral properties and their dependence on redshift and luminosity have led to conflicting conclusions. For example, while Bechtold et al. (2003) reported that the X-ray photon indices $(\Gamma)$ of $z>4$ AGNs are flatter $(1.5 \pm 0.5)$ than those of nearby AGNs, Grupe et al. (2005) reported that their $\Gamma$ are rather steep $(2.23 \pm 0.48)$. The first study was based on a sample of 16 radio-quiet sources observed

\footnotetext{
${ }^{6}$ See http://www.astro.psu.edu/users/niel/papers/highz-xray-detected.dat for a regularly updated compilation of X-ray detections at $z>4$.
} 
TABLE 1

Log of XMM-Newton Observations

\begin{tabular}{|c|c|c|c|c|c|c|c|c|c|}
\hline \multirow[b]{2}{*}{ AGN } & \multirow[b]{2}{*}{$z$} & \multirow[b]{2}{*}{$\alpha(\mathrm{J} 2000.0)$} & \multirow[b]{2}{*}{$\delta(\mathrm{J} 2000.0)$} & \multirow{2}{*}{$\begin{array}{c}\text { GaLACTIC } N_{\mathrm{H}}{ }^{\mathrm{a}} \\
\left(10^{20} \mathrm{~cm}^{-2}\right)\end{array}$} & \multirow[b]{2}{*}{ OBservation Date } & \multicolumn{3}{|c|}{ Exposure Time (ks)/Total Counts } & \multirow[b]{2}{*}{ REFERENCE } \\
\hline & & & & & & MOS1 & MOS2 & $\mathrm{pn}$ & \\
\hline PSS $0121+0347^{\mathrm{b}}$. & 4.13 & 012126.2 & +034706.3 & 3.23 & 2004 Jan 09 & $25.7 / 139$ & $25.9 / 185$ & $21.7 / 540$ & 1 \\
\hline SDSS $0210-0018^{\mathrm{b}}$. & 4.77 & 021043.2 & -001818.3 & 2.66 & 2005 Feb 05 & $18.7 / 112$ & $18.7 / 117$ & $16.5 / 594$ & 1 \\
\hline SDSS $0231-0728 \ldots \ldots \ldots \ldots$ & 5.41 & 023137.7 & -072854.5 & 2.90 & 2004 Jan 07 & $31.6 / 70$ & $31.6 / 101$ & $27.7 / 309$ & 1 \\
\hline \multicolumn{10}{|c|}{ Archival Observations } \\
\hline Q0000-263.......................... & 4.10 & 000322.9 & -260316.8 & 1.67 & 2002 Jun 25 & $34.0 / 295$ & $34.0 / 322$ & $34.0 / 1229$ & 2 \\
\hline BR $0351-1034 \ldots \ldots \ldots \ldots \ldots$ & 4.35 & 035346.9 & -102519.0 & 4.08 & 2002 Aug 23 & $17.9 / 31$ & $17.9 / 15$ & $15.1 / 166$ & 3 \\
\hline SDSS $1030+0524 \ldots \ldots \ldots \ldots$ & 6.28 & 103027.1 & +052455.0 & 3.17 & 2003 May 22 & $44.3 / 80$ & $43.9 / 81$ & $43.4 / 333$ & 4 \\
\hline
\end{tabular}

NoтE.-Units of right ascension are hours, minutes, and seconds, and units of declination are degrees, arcminutes, and arcseconds.

a Galactic absorption column densities from Dickey \& Lockman (1990), obtained using the optical coordinates of the sources given in this table and using the HEASARC $N_{\mathrm{H}}$ tool at http://heasarc.gsfc.nasa.gov/cgi-bin/Tools/w3nh/w3nh.pl.

b Moderately radio-loud source.

RefERENCES.-(1) This work; (2) Ferrero \& Brinkmann 2003; (3) Grupe et al. 2004; (4) Farrah et al. 2004; (5) Grupe et al. 2005.

by Chandra with low total counts (see Table 1 of Bechtold et al. 2003), and one of their objects (SDSS J103027.10+052455.0, hereafter SDSS $1030+0524$ ) is common to the present work. The second study was based on a sample of 16 sources (11 of which are radio-quiet) observed with $X M M-N e w t o n$ with low to high total counts (see Table 1 of Grupe et al. 2005); four of those sources (Q0000-263, BR 0351-1034, SDSS 1030+0524, and BR 2237-0607) are common to the present work, and we use the same X-ray data that were used in that study. The recent analysis by Vignali et al. (2005), which is based on the joint X-ray spectral fitting of a sample of $48 z>4$ radio-quiet AGNs observed with Chandra in the redshift range 3.99-6.28, argues that the X-ray spectral properties of AGNs do not show any significant evolution or luminosity dependence. Our aim in this paper is to address this puzzle by uniformly analyzing a sample of moderate- to high-quality X-ray spectra of $z>4$ AGNs, with $\approx 200-1000$ counts per source. In this paper, we consider radioquiet and radio-loud AGNs separately, as radio-loud sources might exhibit X-ray emission from their jets. The X-ray properties of the two AGN subclasses differ significantly from one another; for example, the X-ray spectra of radio-loud AGNs are flatter than those of radio-quiet sources (e.g., Wilkes \& Elvis 1987; Cappi et al. 1997; Porquet et al. 2004), and, at high redshift, radio-loud AGNs show signs of higher intrinsic absorption (e.g., Worsley et al. 2004a, 2004b) and larger X-ray variability (e.g., Fabian et al. 1999). Since radio-quiet sources represent the majority of luminous AGNs at all redshifts (e.g., Stern et al. 2000; Ivezić et al. 2002; Petric et al. 2003), our analysis focuses on the properties of this subclass.

Moderate- to high-quality spectra of only five $z>4$ radioquiet AGNs have been obtained with XMM-Newton and Chandra over the past three years, with only one such source having more than 1000 counts (Q0000-263; Ferrero \& Brinkmann 2003; see also Farrah et al. 2004; Grupe et al. 2004, 2005; Schwartz \& Virani 2004). In this paper we almost double that number with new data from XMM-Newton, adding two more sources with over 1000 counts, and study the broadband X-ray spectral properties of 10 AGNs with an average redshift of 4.8. In $\S 2$ we describe our five $X M M$-Newton observations, the data reduc- tion, and the analysis. In $\S 3$ we expand our original sample by adding archived high-quality XMM-Newton and Chandra spectra of five $z>4$ radio-quiet AGNs, and in $\S 4$ we investigate the mean X-ray spectral properties of our radio-quiet sample. Our results are discussed in $\S 5$ and summarized in $\S 6$. Throughout the paper we use the standard cosmological model, with parameters $\Omega_{\Lambda}=0.7, \Omega_{M}=0.3$, and $H_{0}=70 \mathrm{~km} \mathrm{~s}^{-1} \mathrm{Mpc}^{-1}$ (Spergel et al. 2003).

\section{NEW HIGH-QUALITY SPECTRA OF $z>4$ AGNs}

\subsection{Observations and Data Reduction}

We have obtained imaging spectroscopic observations of five $z>4$ AGNs with XMM-Newton (Jansen et al. 2001). Three of the sources were discovered in the DPOSS survey and were detected in X-rays by Vignali et al. (2003a); two were discovered in the SDSS survey and detected in X-rays by Vignali et al. (2001, 2003b). Three of the sources are radio-quiet with an upper limit on the radio flux, while two, PSS $0121+0347$ and SDSS 0210-0018, are moderately radio-loud, following the classification of Kellermann et al. (1989), and have $R=300$ and 80, respectively (Vignali et al. 2001, 2003a). These five sources were among the X-ray brightest of a sample of $z>4$ AGNs previously targeted with Chandra snapshot ( $\lesssim 10 \mathrm{ks}$ ) observations (Vignali et al. 2001, 2003a, 2003b). One of these sources, SDSS $0231-0728$ at $z=5.41$, is the highest redshift AGN detected with the automated SDSS detection algorithm (Anderson et al. 2001). The short, higher spatial resolution Chandra observations allow the prediction of the number of counts expected from the XMM-Newton observations, and they eliminate the possibility of significant source confusion due to the lower spatial resolution of XMM-Newton. No nearby contaminating sources were detected in the Chandra images of our five AGNs.

A log of the new XMM-Newton observations appears in Table 1 (first five sources). The data were processed using standard $\mathrm{SAS}^{7}$ version 6.1.0 and FTOOLS tasks. The event files of all the

${ }^{7}$ XMM-Newton Science Analysis System. See http://xmm.vilspa.esa.es/ external/xmm_sw_cal/sas_frame.shtml. 
TABLE 2

Best-Fit X-Ray Spectral Parameters

\begin{tabular}{|c|c|c|c|c|}
\hline AGN & $\begin{array}{c}N_{\mathrm{H}}^{\mathrm{a}} \\
\left(10^{22} \mathrm{~cm}^{-2}\right)\end{array}$ & $\Gamma$ & $\begin{array}{c}f_{E}(1 \mathrm{keV})^{\mathrm{b}} \\
\left(10^{-6} \mathrm{keV} \mathrm{cm}^{-2} \mathrm{~s}^{-1} \mathrm{keV}^{-1}\right)\end{array}$ & $\chi^{2}(\mathrm{DOF})$ \\
\hline \multicolumn{5}{|c|}{ New Observations } \\
\hline 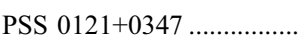 & $\leq 2.91$ & $1.81 \pm 0.16$ & $7.64_{-1.01}^{+0.96}$ & $54.8(52)$ \\
\hline SDSS $0210-0018 \ldots \ldots \ldots \ldots . . . .$. & $\leq 4.17$ & $1.81_{-0.14}^{+0.15}$ & $6.45 \pm 0.78$ & $64.2(75)$ \\
\hline SDSS $0231-0728 \ldots \ldots \ldots \ldots$ & $\leq \overline{1} 9.90$ & $1.85_{-0.31}^{+0.33}$ & $2.10_{-0.58}^{+0.59}$ & $25.3(43)$ \\
\hline PSS $0926+3055 \ldots \ldots \ldots \ldots \ldots$ & $\leq 1.02$ & $1.99 \pm 0.08$ & $17.58 \pm 1.15$ & 76.1(78) \\
\hline PSS $1326+0743 \ldots \ldots \ldots \ldots \ldots$ & $\leq 0.47$ & $1.87 \pm 0.10$ & $12.54_{-1.13}^{+0.84}$ & $51.2(65)$ \\
\hline \multicolumn{5}{|c|}{ Archival Observations } \\
\hline Q0000-263......................... & $\leq 0.40$ & $2.02 \pm 0.07$ & $12.59 \pm 0.75$ & $71.3(82)$ \\
\hline BR $0351-1034 \ldots \ldots \ldots \ldots \ldots . . . . . .$. & $\leq 5.29$ & $1.76_{-0.26}^{+0.31}$ & $5.24_{-0.97}^{+0.96}$ & $8.7(10)$ \\
\hline SDSS $1030+0524 \ldots \ldots \ldots \ldots$ & $\leq 7.77$ & $1.92_{-0.21}^{+0.23}$ & $1.39 \pm 0.23$ & $40.9(44)$ \\
\hline BR 2237-0607..................... & $\leq 3.30$ & $2.09_{-0.21}^{+0.22}$ & $3.82_{-0.57}^{+0.59}$ & $20.4(25)$ \\
\hline
\end{tabular}

Note.-The best-fit photon index, normalization, and $\chi^{2}$ were obtained from a model consisting of a Galactic-absorbed power law without any intrinsic absorption.

${ }^{a}$ Intrinsic column density. Upper limits were calculated using the intrinsically absorbed power-law model with Galactic absorption, and they represent $90 \%$ confidence limits for each value, taking one parameter of interest $\left(\Delta \chi^{2}=2.71\right.$; e.g., Avni 1976).

b Power-law normalization for the pn data, taken from joint fitting of all three EPIC detectors with the Galacticabsorbed power-law model.

observations were filtered to include events with $f l a g=0$, and pattern $\leq 12$ (pattern $\leq 4)$ and $200 \leq \mathrm{pi} \leq 12,000(150 \leq$ pi $\leq 15,000)$ for the $\operatorname{MOS}$ ( $p n$ ) detectors. The event files of the observations of SDSS 0210-0018, SDSS 0231-0728, and PSS $0926+3055$ were also filtered to remove periods of flaring activity, which were apparent in the light curve of the entire fullframe window. Such flaring activity, which originates in the Earth's magnetosphere, depends on several unpredictable factors, and it can significantly reduce the good-time intervals of an observation. ${ }^{8}$ The observations of SDSS $0210-0018$, SDSS 0231-0728, and PSS $0926+3055$ were shortened by 16,10, and $0.6 \mathrm{ks}$, respectively, as a result of this filtering. The event files of the PSS $0121+0347$ and PSS $1326+0743$ observations were not filtered in time, since the entire observation of the first source was performed during a moderate background flare period with count rates $\sim 3$ times higher than nominal values, and the fullframe count rate light curve of the second source did not show any flaring. The exposure times listed in Table 1 reflect the filtered data used in the analysis.

We searched for X-ray emission from an additional radio-quiet AGN, SDSS J021102.72-000910.3 at $z=4.90$ (Fan et al. 1999), which lies close to the edge of the field of view in the $X M M$ Newton observation of SDSS $0210-0018$. The former source is not detected, and the $3 \sigma$ detection threshold is $\sim 120$ counts. Using PIMMS, ${ }^{9}$ this translates to an upper limit on the unabsorbed observed-frame $0.5-2 \mathrm{keV}$ flux of $\lesssim 10^{-14} \mathrm{ergs} \mathrm{cm}^{-2} \mathrm{~s}^{-1}$, which is consistent with the $3.1_{-1.4}^{+2.2} \times 10^{-15} \mathrm{ergs} \mathrm{cm}^{-2} \mathrm{~s}^{-1}$ flux measured by Vignali et al. (2001).

\subsection{Spectral Analysis}

To extract X-ray spectra, we used a source extraction aperture radius of $30^{\prime \prime}$ in the images of all three European Pho-

\footnotetext{
${ }^{8}$ On average, $\sim 35 \%$ of the data from $X M M$-Newton observations with exposure times greater than $10 \mathrm{ks}$ are lost due to flaring (K. Kuntz 2005, private communication). For more details see the XMM-Newton User Handbook at http://xmm .vilspa.esa.es/external/xmm_user_support/documentation/uhb/index.html.

${ }_{9}$ Portable Interactive Multi-Mission Simulator at http://heasarc.gsfc.nasa .gov/Tools/w3pimms.html.
}

ton Imaging Camera (EPIC) detectors for all but one AGN, PSS 0121+0347. For this source we used an aperture radius of $20^{\prime \prime}$ for the source extraction, since a larger aperture was affected by the (mainly hard X-ray) background flare, resulting in a hardening of the spectrum and a lowering of the signalto-noise ratio. For a nominal background level, increasing the aperture radius from $20^{\prime \prime}$ to $30^{\prime \prime}$ should not have resulted in any noticeable change in either photon index or normalization (Kirsch et al. 2004). Background regions were taken to be at least as large as the source regions; these were annuli when there were no neighboring sources and circles otherwise. The redistribution matrix files (RMFs; which include information on the detector gain and energy resolution) and the ancillary response files (ARFs; which include information on the effective area of the instrument, filter transmission, detector window transmission and efficiency, and any additional energy-dependent effects) for the spectra were created with the SAS tasks RMFGEN and ARFGEN, respectively. ${ }^{10}$ We note that the two reflection grating spectrometer detectors on XMM-Newton do not have sufficient counts to perform a high-resolution spectral analysis for any of our sources.

The spectra were grouped with a minimum of $10-25$ photons per bin using the task GRPPHA. For each AGN we used XSPEC version 11.3.0 (Arnaud 1996) to fit the data with the following models: (1) a power-law model and a Galactic absorption component (Dickey \& Lockman 1990), which was kept fixed during the fit; and (2) a model similar to the first with an added intrinsic (redshifted) neutral absorption component. In all the fits we assumed solar abundances (Anders \& Grevesse 1989) and used the PHABS absorption model in XSPEC with the Balucinska-Church \& McCammon (1992) cross sections. We fitted the data from each EPIC detector alone, jointly fitted the data of the two MOS detectors, and jointly fitted all three EPIC detectors with these models. In general, the five different fitting results were consistent with each other within the uncertainties,

${ }^{10}$ For more details see the NASA/GSFC calibration memo CAL/GEN/92-002 at http://heasarc.gsfc.nasa.gov/docs/heasarc/caldb/docs/memos/cal_gen_92_002/ cal_gen_92_002.html. 


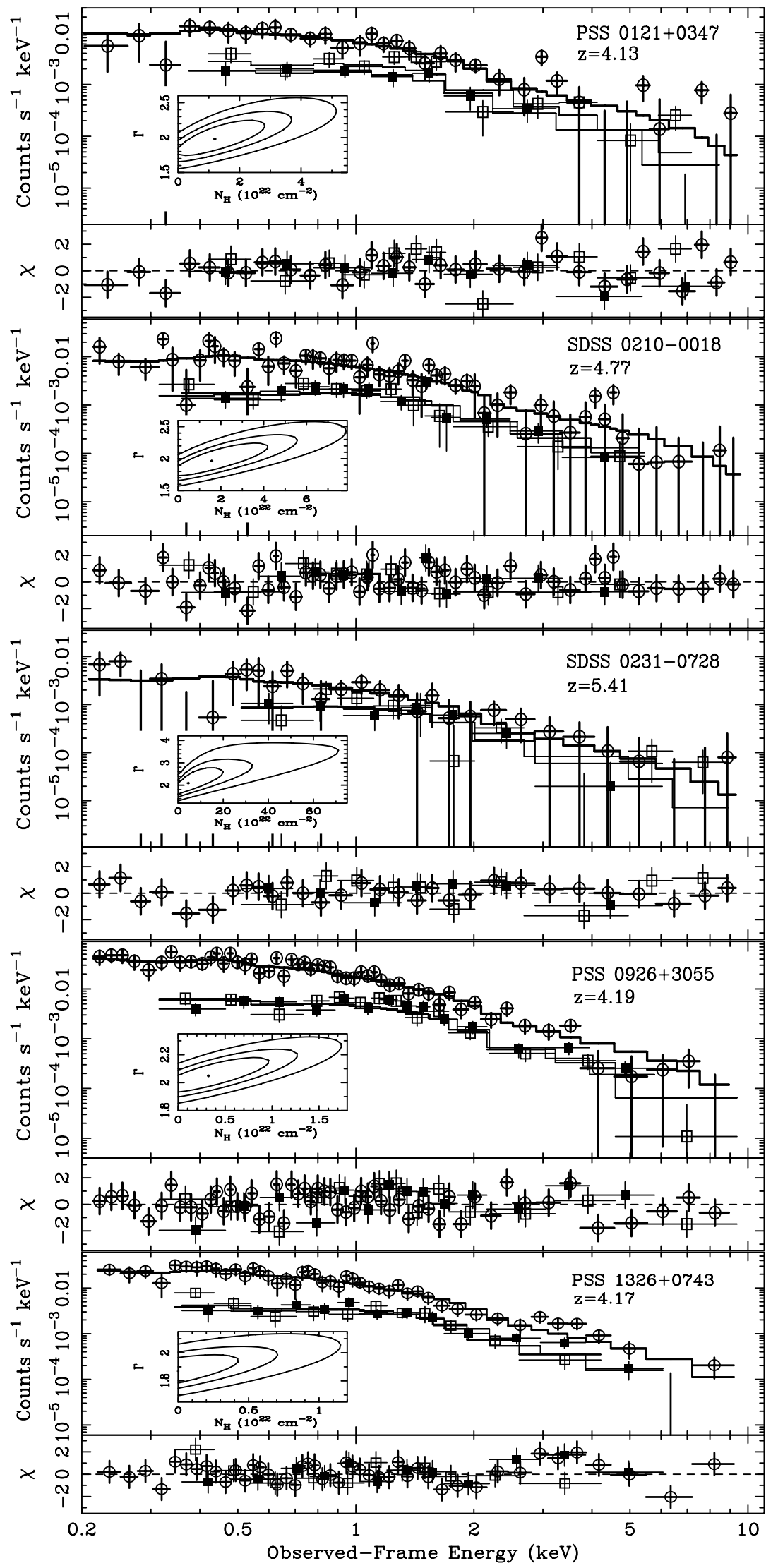

Fig. 1. - Data, best-fit spectra, and residuals for our new XMM-Newton observations of five $z>4$ AGNs. Open circles, filled squares, and open squares represent the pn, MOS1, and MOS2 data, respectively. Solid lines represent the best-fit model for each spectrum, and the thick line marks the best-fit model for the pn data. The $\chi$ residuals are in units of $\sigma$ with error bars of size 1 . The inset in each panel shows $68 \%, 90 \%$, and $99 \%$ confidence contours for the intrinsic absorption $\left(N_{\mathrm{H}}\right)$ and photon index $(\Gamma)$. 
TABLE 3

FluXes, Luminosities, and $\alpha_{\text {ox }}$ For the Sample of $z>4$ AGNs

\begin{tabular}{|c|c|c|c|c|c|c|c|c|}
\hline AGN & $\mathrm{AB}_{1450(1+z) \AA}$ & $M_{B}$ & $\begin{array}{c}f_{\nu}[2500(1+z) \AA]^{\mathrm{a}} \\
(\mathrm{mJy})\end{array}$ & $\begin{array}{l}\log \nu L_{\nu}(2500 \AA) \\
\left(\operatorname{ergs~s}^{-1}\right)\end{array}$ & $\begin{array}{c}\text { Optical } \\
\text { Reference }\end{array}$ & $\begin{array}{c}F_{0.5-2 \mathrm{keV}^{\mathrm{b}}} \\
\left(10^{-15} \mathrm{ergs} \mathrm{cm}^{-2} \mathrm{~s}^{-1}\right)\end{array}$ & $\begin{array}{l}\log L_{2-10 \mathrm{keV}} \\
\left(\mathrm{ergs} \mathrm{s}^{-1}\right)\end{array}$ & $\alpha_{\mathrm{ox}}$ \\
\hline \multicolumn{9}{|c|}{ New Observations } \\
\hline PSS $0121+0347 \ldots \ldots \ldots \ldots$ & 18.5 & -28.3 & $0.222 \pm 0.071$ & 46.94 & 1 & $17.02_{-2.28}^{+2.19}$ & 45.51 & $-1.65 \pm 0.03$ \\
\hline SDSS $0210-0018 \ldots \ldots \ldots$ & 19.3 & -27.7 & $0.106 \pm 0.011$ & 46.71 & 2 & $14.37_{-1.76}^{+1.77}$ & 45.27 & $-1.54_{-0.02}^{+0.03}$ \\
\hline SDSS $0231-0728 \ldots \ldots \ldots$ & 19.3 & -27.9 & $0.062 \pm 0.030$ & 46.56 & 3 & $4.68_{-1.29}^{+1.35}$ & 45.21 & $-1.62 \pm 0.06$ \\
\hline PSS $0926+3055 \ldots \ldots \ldots \ldots$. & 16.7 & -30.1 & $1.167 \pm 0.042$ & 47.67 & 1 & $39.04_{-2.54}^{+2.57}$ & 45.89 & $-1.76_{-0.01}^{+0.03}$ \\
\hline \multicolumn{9}{|c|}{ Archival Observations } \\
\hline Q0000-263..................... & 17.5 & -29.3 & $0.579 \pm 0.058$ & 47.35 & 4 & $12.59 \pm 0.75$ & 45.72 & $-1.70_{-0.01}^{+0.03}$ \\
\hline BR $0351-1034 \ldots \ldots \ldots \ldots . .$. & $18.7^{\mathrm{c}}$ & -28.2 & $0.185 \pm 0.019$ & 46.89 & 5 & $11.69_{-2.19}^{+2.22}$ & 45.39 & $-1.69 \pm 0.04$ \\
\hline SDSS $1030+0524 \ldots \ldots \ldots$ & 19.7 & -27.8 & $0.076 \pm 0.008$ & 46.75 & 6 & $3.09_{-0.52}^{+0.53}$ & 45.18 & $-1.69 \pm 0.04$ \\
\hline BR $2237-0607 \ldots \ldots \ldots \ldots . . .$. & $18.3^{\mathrm{c}}$ & -28.5 & $0.267 \pm 0.027$ & 47.08 & 5 & $8.50_{-1.27}^{+1.32}$ & 45.32 & $-1.74_{-0.03}^{+0.04}$ \\
\hline
\end{tabular}

\footnotetext{
${ }^{\mathrm{a}}$ Assuming a UV continuum of the form $f_{\nu} \propto \nu^{-0.79}$ (Fan et al. 2001).

b Galactic absorption-corrected flux in the observed $0.5-2 \mathrm{keV}$ band.

c Estimated using an empirical relation between the Automatic Plate Measuring Facility Survey $R$ and $\mathrm{AB}_{1450(1+z) \AA}$ magnitudes and redshift; see $\S 2$ of Kaspi et al. (2000).
}

References.-(1) Vignali et al. 2003a; (2) Vignali et al. 2001; (3) Vignali et al. 2003b; (4) Schneider et al. 1989; (5) Storrie-Lombardi et al. 1996; (6) Brandt et al. 2002.

and the joint fit of all three EPIC detectors had the smallest uncertainty on the spectral parameters. The best-fit results for the joint fit of the three EPIC detectors are summarized in Table 2 (first five sources). For all five sources the best-fit model is a Galactic-absorbed power law; an $F$-test carried out on the data does not warrant the addition of an intrinsic absorber in any of the spectra. The $90 \%$ confidence upper limits on intrinsic $N_{\mathrm{H}}$ appear in Table 2. The spectra of the five AGNs and their best-fit models appear in Figure 1. Also shown for each AGN is a confidence contour plot of the $\Gamma-N_{\mathrm{H}}$ parameter space. No strong systematic residuals are seen for any of the fits. Optical fluxes and luminosities, and X-ray fluxes and luminosities of the sources computed using the best-fit spectral parameters of Table 2, appear in Table 3 (first five sources).

\subsection{Serendipitous Sources}

Since the luminous AGNs under study are probably associated with massive potential wells in the earliest collapsed large-scale structures, we searched for candidate $z>4$ AGNs in the XMMNewton images of three of our sources with SDSS imaging (SDSS 0210-0018, SDSS 0231-0728, and PSS 1326+0743) in order to look for AGN clustering on scales of $\approx 0.1-5 \mathrm{Mpc}$ at $z \sim 4-5$ (e.g., see Djorgovski et al. 2003; Vignali et al. 2003b; Hennawi et al. 2005). We matched X-ray and SDSS sources in those fields and utilized the Richards et al. (2002) color criteria, as well as a pointlike morphology criterion, to identify $\approx 3 \mathrm{X}$-rayemitting $z \gtrsim 4$ AGN candidates for future optical spectroscopy.

\section{ARCHIVAL SAMPLE OF $z>4$ RADIO-QUIET AGNs}

To improve the statistics on X-ray spectral properties, we expanded the sample by adding moderate- to high-quality data of five radio-quiet $z>4 \mathrm{AGNs}$, obtained from the XMM-Newton and Chandra archives. A minimum of $\sim 200(\sim 100)$ photons per source from XMM-Newton (Chandra) observations were considered useful to our analysis. The observation log of the four sources observed by XMM-Newton appears in Table 1, which also gives the references to the original papers presenting the results of those observations. We reduced the observation data files of these sources following the analysis procedures described in $\S 2$. As in the case of our original sample, we were only able to place upper limits on the intrinsic column densities in these sources. The best-fit model parameters for the X-ray spectra of these sources are listed in Table 2.

The fifth observation, a $118 \mathrm{ks}$ Chandra ACIS-S observation of SDSS J130608.26+035626.3 (hereafter SDSS 1306+0356) at $z=5.99$ on 2003 November 29-30, was reduced and reanalyzed following the same source and background extraction parameters as those used in the original paper reporting that observation (Schwartz \& Virani 2004) applying standard CIAO ${ }^{11}$ version 3.2 routines to the data. The events were grouped into bins that had a minimum of 15 counts per bin, and the model fit to the data included a constant Galactic absorption component $\left(N_{\mathrm{H}}=2.07 \times 10^{20} \mathrm{~cm}^{-2}\right)$, a power-law component, and a neutral $\mathrm{Fe} \mathrm{K} \alpha$ line at rest-frame $6.4 \mathrm{keV}$ with a width of $\sigma=0.1 \mathrm{keV}$, since there is a feature in the spectrum at that location hinting at the presence of such a line (see Schwartz \& Virani 2004). The best-fit photon index is $\Gamma=1.82_{-0.31}^{+0.29}$, the flux normalization at $1 \mathrm{keV}$ is $(1.23 \pm 0.24) \times 10^{-6} \mathrm{keV} \mathrm{cm}^{-2} \mathrm{~s}^{-1} \mathrm{keV}^{-1}$, and the $\mathrm{Fe} \mathrm{K} \alpha$ rest-frame equivalent width is $1.0_{-0.8}^{+1.1} \mathrm{keV}$.

The optical fluxes and luminosities, and X-ray fluxes and luminosities of the five sources, based on the best-fit spectral parameters of Table 2 and of the single Chandra observation, are listed in Table 3. Plots of the spectra and best-fit models, which are generally consistent with our results, appear in the original papers cited in Table 1 and in Schwartz \& Virani (2004) for the single Chandra observation.

\section{JOINT SPECTRAL FITTING OF $z>4$ RADIO-QUIET AGNs}

To derive the best possible constraints on the average X-ray spectral properties of radio-quiet $z>4$ AGNs, we jointly fitted the spectra of the expanded sample of eight radio-quiet AGNs (e.g., see $\S 6$ of Vignali et al. 2005). We combined the spectra from all the detectors of the observations of the eight AGNs, 22 data sets in total, and used XSPEC to fit their spectra jointly. We

\footnotetext{
${ }^{11}$ Chandra Interactive Analysis of Observations. See http://asc.harvard .edu/ciao.
} 
TABLE 4

Best-Fit Parameters from Joint Fitting of Eight Radio-Quiet $z>4$ AGNs

\begin{tabular}{|c|c|c|c|c|c|c|}
\hline Spectral Model: Galactic-Absorbed Power Law and & Energy Range $^{\mathrm{a}}$ & $\begin{array}{c}N_{\mathrm{H}} \\
\left(10^{22} \mathrm{~cm}^{-2}\right)\end{array}$ & $\Gamma$ & $\begin{array}{c}\mathrm{EW}(\mathrm{Fe} \mathrm{K} \alpha)^{\mathrm{b}} \\
(\mathrm{eV})\end{array}$ & $R^{\mathrm{c}}$ & $\chi^{2}(\mathrm{DOF})$ \\
\hline \multirow[t]{2}{*}{ 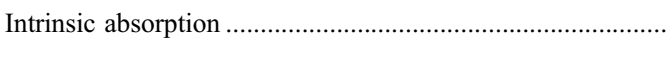 } & $\mathrm{E}$ & $\leq 0.28$ & $1.97_{-0.04}^{+0.06}$ & $\cdots$ & $\ldots$ & $308.3(360)$ \\
\hline & $\mathrm{C}$ & $\leq 0.84$ & $2.02_{-0.06}^{+0.04}$ & $\ldots$ & $\ldots$ & $291.3(338)$ \\
\hline \multirow[t]{2}{*}{ 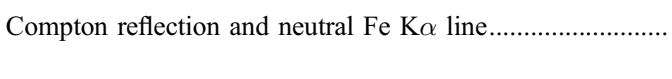 } & $\mathrm{E}$ & $\ldots$ & $1.98_{-0.09}^{+0.10}$ & $\leq 189$ & $\leq 1.22$ & $306.0(359)$ \\
\hline & $\mathrm{C}$ & $\ldots$ & $1.95_{-0.08}^{+0.10}$ & $\leq 191$ & $\leq 1.03$ & $291.2(337)$ \\
\hline
\end{tabular}

${ }^{a} \mathrm{E}$ : entire energy range of all the spectra. $\mathrm{C}$ : the common rest-frame energy range among all sources $(1.5-51 \mathrm{keV})$.

b Rest-frame equivalent width calculated with a mean redshift of 4.8 .

c Compton hump relative reflection parameter (see Magdziarz \& Zdziarski 1995).

fit two different models: (1) a power law that includes both Galactic and intrinsic absorption and (2) a power law with Galactic absorption including a Compton reflection component, using the pexrav model in XSPEC (Magdziarz \& Zdziarski 1995), and a neutral narrow Gaussian Fe K $\alpha$ line. The Compton reflection component, also known as a Compton reflection "hump," is the spectral manifestation of hard X-ray photons emitted in a corona of hot electrons and reflected off the relatively colder accretion disk (e.g., see $\S 3.5$ of Reynolds \& Nowak 2003 and references therein). The Compton reflection hump feature is observed in the rest-frame $\sim 7-60 \mathrm{keV}$ energy range, peaking at rest-frame $\sim 30 \mathrm{keV}$. The joint fitting process was carried out twice for both models: first, to include the entire energy range of all the data sets, and a second time to include only the common rest-frame energy range of the eight sources: $1.5 \mathrm{keV} \lesssim E_{\text {rest }} \lesssim 51 \mathrm{keV}$. The total number of photons used in the joint fitting process is $\sim 7000$ ( $\sim 6400$ for the common energy range), and it is larger by an order of magnitude than the number of counts used in the joint fitting of $48 z>4$ radio-quiet AGNs by Vignali et al. (2005).

The fitting process with the first model required that all data sets be fitted with a single power law and a single intrinsic absorption column. Therefore, the photon index and intrinsic absorption column were each tied to a single value across all data sets, and these two parameters were allowed to vary jointly (see the discussion of intrinsic dispersion in $\S 5.1$ as a partial justification of

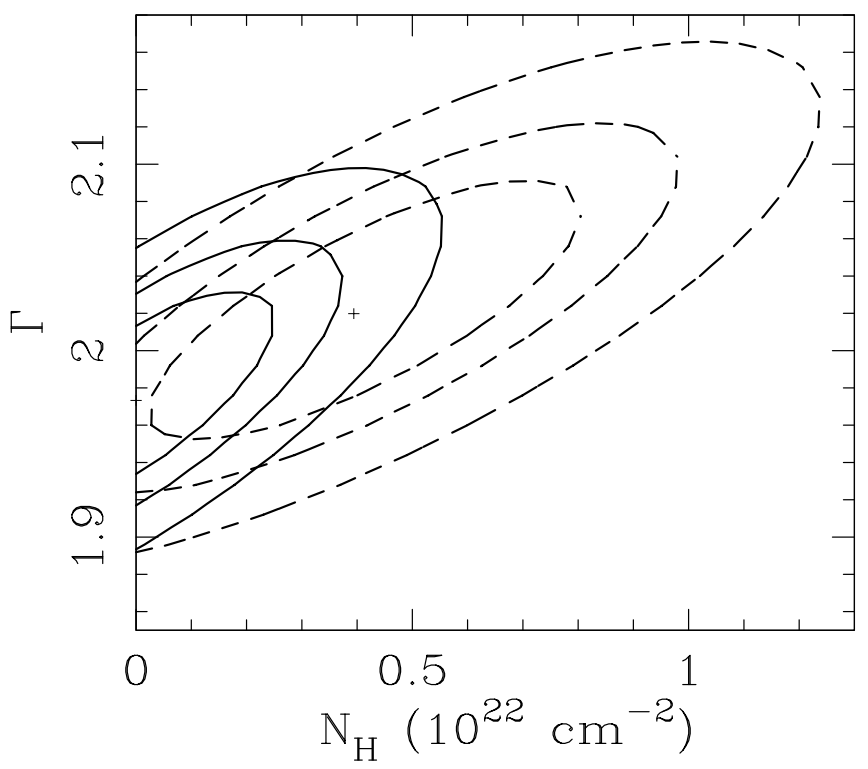

FIG. 2.-68\%, 90\%, and $99 \%$ confidence regions for the photon index vs. intrinsic column density derived from joint spectral fitting of our sample of eight radio-quiet AGNs. Solid (dashed) contours refer to the entire (common) energy range of the AGNs (see $\S 4$ for more details). this approach). The power-law normalizations of all data sets were allowed to vary freely. For the second model we used a single power law and a single relative-reflection component $(R$, where $R$ is defined as $\Omega / 2 \pi$ and $\Omega$ is the solid angle subtended by the reflecting medium) of the Compton hump for all data sets and allowed them to vary jointly. We fixed the rest-frame energy and width $(\sigma)$ of the neutral Fe $\mathrm{K} \alpha$ line to 6.4 and $0.1 \mathrm{keV}$, respectively, and tied all the power-law and Gaussian normalizations to two different values, allowing them to vary jointly; each data set was assigned a constant scaling factor to account for the different flux levels of each AGN and each detector. The best-fit values and upper limits for the parameters used in the joint fits are given in Table 4. Figure 2 is a contour plot of $\Gamma$ versus $N_{\mathrm{H}}$ as a result of the joint fitting process using the first model. The noticeable difference between the confidence contours in the entire and common energy ranges stems from the fact that the two AGNs at $z \simeq 6$ increase the low-energy threshold in the common energy range, and as a consequence many low-energy photons from the X-ray-bright AGNs at $z \simeq 4.2$ are ignored in the fit. This causes the upper limit on the intrinsic absorption in the common energy range to rise significantly relative to the upper limit when using the entire energy range.

We repeated the joint fitting process with the first model and fitted the four most optically luminous radio-quiet sources separately from the four least optically luminous radio-quiet sources for both the entire and common energy ranges of the two subsamples. Both $\Gamma$ and $N_{\mathrm{H}}$ in the two luminosity groups are consistent with one another and with the values we obtain by fitting the entire radio-quiet sample. For the fitting using the entire energy range, we measure $\Gamma=1.99 \pm 0.06$ for the most luminous sources and $\Gamma=1.95_{-0.20}^{+0.23}$ for the least luminous sources.

\section{RESULTS AND DISCUSSION \\ 5.1. X-Ray Photon Index}

The photon indices and their uncertainties for the full sample of $10 \mathrm{AGNs}$, in the $\sim 1-50 \mathrm{keV}$ rest-frame energy range (the range where most photons are detected), are listed in Table 2. A statistical summary of our expanded sample's photon index

TABLE 5

Рhoton Index Statistics of $z>4$ AGNs

\begin{tabular}{|c|c|c|}
\hline \multirow[b]{2}{*}{ Statistic } & \multicolumn{2}{|c|}{ РнотоN Index $(\Gamma)$} \\
\hline & Entire Sample & Radio-Quiet Sources \\
\hline Unweighted mean & 1.89 & 1.92 \\
\hline Unweighted standard deviation ........ & 0.11 & 0.11 \\
\hline Unweighted error of the mean ......... & 0.03 & 0.04 \\
\hline Counts-weighted mean ……................ & 1.93 & 1.95 \\
\hline Median & 1.86 & 1.90 \\
\hline
\end{tabular}




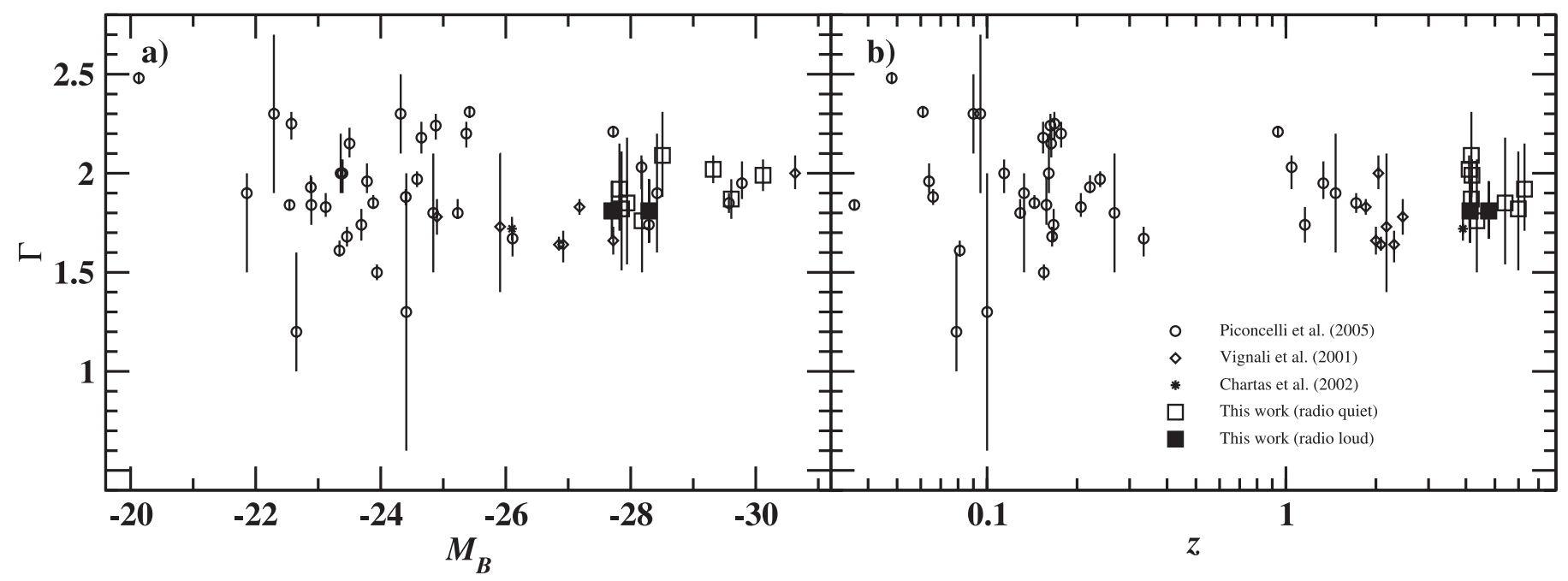

FIG. 3.-X-ray photon index ( $\Gamma$ ) vs. ( $a$ ) absolute $B$ magnitude and $(b)$ redshift, adapted from Vignali et al. (2005). The eight (two) radio-quiet (moderately radio-loud) AGNs presented in this work are marked with open (filled) squares. Note that the photon index does not show any clear dependence on either luminosity or redshift.

distribution appears in Table 5. Using the maximum likelihood method of Maccacaro et al. (1988) we find no significant intrinsic dispersion in $\Gamma$; this is expected, since the individual uncertainties on $\Gamma$ are larger than the spread in individual $\Gamma$ values. In Figure 3 we plot $\Gamma$ versus absolute $B$ magnitude and redshift for our expanded sample, together with previous measurements of the photon index in other AGN samples ( $\operatorname{see} \S 6$ of Vignali et al. 2005 and references therein). There is no obvious systematic difference between the photon indices of our radioquiet sources and those of luminous AGNs observed at lower redshifts (e.g., the Piconcelli et al. 2005 sample of nearby AGNs). Using a Spearman rank correlation method we find no significant correlations between $\Gamma$ in radio-quiet sources and either luminosity or redshift as also found at lower redshift $(z \lesssim 0.4)$ by, e.g., Porquet et al. (2004). Applying a linear regression algorithm that accounts for intrinsic scatter and measurement errors (Akritas \& Bershady 1996), we obtain $|\partial \Gamma / \partial z|<0.04$ for the 51 radio-quiet AGNs plotted in Figure 3. From the joint fitting of eight radioquiet AGN spectra using the absorbed power-law model we find $\Gamma=1.97_{-0.04}^{+0.06}$. This is consistent with both the unweighted mean of the individual $\Gamma$ values and the counts-weighted mean $\Gamma$ for the radio-quiet sample.

The mean photon indices we find for $z>4$ radio-quiet AGNs are also all consistent with those calculated by Vignali et al. (2005). All these photon index values confirm and strengthen the Vignali et al. (2005) result that the X-ray photon index does not depend on optical luminosity or redshift. In particular, our results do not support either of the reports for a significantly different photon index at low versus high redshift (Bechtold et al. 2003; Grupe et al. 2005). Bechtold et al. (2003) used a heterogeneous sample of low-redshift Röntgensatellit (ROSAT) sources and high-redshift sources detected with Chandra. The mix of different data sets may have produced a bias that led Bechtold et al. (2003) to report a flattening of $\Gamma$ at higher redshift (e.g., due to spectral curvature at low energies and mission-to-mission crosscalibration uncertainties). In addition, the counts-weighted mean $\Gamma$ of their $z>4$ Chandra sources is $1.50 \pm 0.15$ (see their Table 2). This is significantly lower than the average $\Gamma$ of $1.93_{-0.09}^{+0.10}$ for 48 radio-quiet AGNs, including the Bechtold et al. (2003) sources, obtained by Vignali et al. (2005). Comparing the photon indices of our four sources that overlap with those of Grupe et al. (2005) shows that in three cases the values are in good agreement. However, for SDSS $1030+0524$ the $\Gamma=2.65 \pm 0.34$ found by Grupe et al. (2005) is significantly steeper and is inconsistent with both the value we obtain, $\Gamma=1.92_{-0.21}^{+0.22}$, and that obtained by Farrah et al. (2004), $\Gamma=2.12 \pm 0.11$. The photon index that we obtain for SDSS $1030+0524$, while slightly harder, is compatible within the errors with the one found by Farrah et al. (2004). By inspection of Tables 1 and 4 of Grupe et al. (2005) it is also apparent that the relatively steep mean $\Gamma$ that they report for their radio-quiet sources, $2.23 \pm 0.48$, is an unweighted mean dominated by sources with limited photon statistics $(\approx 50$ counts), which in most cases result in best-fit $\Gamma$ exceeding a value of 2.2 .

The insignificant dispersion in individual $\Gamma$ values for our $z>4$ AGN sample may be partially driven by a selection bias, since we specifically targeted some of the most X-ray-luminous AGNs known. However, observing down the AGN luminosity function at $z>4$ is challenging with existing X-ray observatories. In fact, the faintest X-ray sources at those redshifts with enough counts to allow meaningful measurement of the photon index were detected in the 2 Ms Chandra Deep Field-North survey (Vignali et al. 2002; see Table 1 of Brandt et al. 2005 for a list of X-ray-faint AGNs at $z>4$ ). The best-studied source in that survey, CXOHDFN J123647.9+620941 at $z=5.19$ and with $L_{2-10 \mathrm{keV}}=10^{44.0} \mathrm{ergs} \mathrm{s}^{-1}$, has $\Gamma=1.8 \pm 0.3$, which is consistent with the photon indices that we find for our sources (Vignali et al. 2002). The photon indices of our least luminous sources do not appear to be significantly different from those of the more luminous ones both individually and on average by splitting the joint spectral fit into two luminosity groups (see $\S 4)$. Inspection of Table 4 of Vignali et al. (2005) also does not show a strong dependence of $\Gamma$ on optical luminosity. In spite of being extremely luminous, our $z>4$ AGNs are otherwise representative of the AGN population by having "normal" restframe ultraviolet (UV) properties (i.e., no broad absorption lines, typical emission-line properties and rest-frame UV continua; Vignali et al. 2001, 2003a, 2003b). Recent multiwavelength observations of $z>4$ AGNs have found that their overall spectral energy distributions (SEDs) are not significantly different from those of lower redshift sources, implying no SED evolution (e.g., see Carilli et al. 2001 and Petric et al. 2003 for radio observations; Pentericci et al. 2003 and Vanden Berk et al. 2004 for UV-optical observations). We argue that the broadband $\mathrm{X}$-ray spectral properties of $z>4$ AGNs are also not significantly different from those of lower redshift sources. 


\subsection{Intrinsic Absorption}

In all the individual fits of the $z>4$ AGNs, we obtained an intrinsic $N_{\mathrm{H}}$ consistent with zero with $90 \%$ confidence. Table 2 lists the upper limits on the intrinsic absorption; they are all in the range $\approx 10^{22}-10^{23} \mathrm{~cm}^{-2}$. The joint fitting procedure yielded an upper limit on the common intrinsic $N_{\mathrm{H}}$ of $\sim(3-8) \times$ $10^{21} \mathrm{~cm}^{-2}$ (Table 4 and Fig. 2). These constraints on intrinsic absorption are the tightest presented to date for $z>4$ AGNs, and our individual fitting results are less prone to bias than the joint fitting results that have been predominantly utilized previously. ${ }^{12}$ Our results show that radio-quiet AGNs at $z>4$ do not appear to have higher absorption columns than their lower redshift counterparts. The metallicity in the inner regions of high-redshift AGNs may be higher, on average, than in their lower redshift counterparts mainly due to their higher luminosities (e.g., Dietrich et al. 2003). Since X-ray photoelectric absorption is predominantly due to metals and not hydrogen, the inferred intrinsic neutral hydrogen column density depends on the assumed metallicity. Therefore, $N_{\mathrm{H}}$ values are inversely proportional to the assumed metallicity, and the quoted upper limits on the best-fit intrinsic columns given in Table 4 are conservative values (as solar abundances were assumed).

Despite the considerable large-scale evolution the universe has experienced since $z \approx 6$, and in particular the strong evolution in the AGN population (e.g., Fan et al. 2003; Croom et al. 2004; Barger et al. 2005; Brandt \& Hasinger 2005), we do not detect significant changes in the central regions of luminous radio-quiet AGNs. Since the host galaxies of AGNs at high redshifts are still in the formation process, one might expect large amounts of gas and dust in the interstellar medium of these hosts that could increase the amount of line-of-sight absorption. Signatures of large amounts of interstellar molecular gas and dust have been recently detected in some of the most distant AGNs (e.g., Carilli et al. 2001; Bertoldi et al. 2003; Walter et al. 2003; Maiolino et al. 2004). In addition, the fact that radio-loud AGNs show signs of increased intrinsic absorption with redshift (see $\S 1$ ) makes it of interest to check whether there is a similar trend for the radio-quiet population. Our results do not show significant intrinsic absorption in any of the $z>4$ radioquiet AGNs, which is in broad agreement with, e.g., the Laor et al. (1997) results for local radio-quiet AGNs. We caution that our AGN sample is far from being complete or representative of the entire AGN population. We have specifically considered only optically and X-ray-bright, radio-quiet AGNs with no broad absorption lines. Steffen et al. (2003) have found that the fraction of type 1 (i.e., unobscured) AGNs increases with X-ray luminosity, suggesting luminosity-dependent absorption. It is therefore possible that the relatively lower intrinsic absorption in our sources is a consequence of them being among the most luminous AGNs known.

\subsection{Fe $\mathrm{K} \alpha$ Line and Compton Reflection}

The joint fitting process placed respectable constraints on the strength of a putative neutral narrow $\mathrm{Fe} \mathrm{K} \alpha$ line; a $90 \%$ confidence upper limit on the rest-frame equivalent width is $\sim 190 \mathrm{eV}$. We also constrained the relative reflection component of a Compton

\footnotetext{
12 Since photoelectric absorption by neutral gas is an extremely strong spectral effect, a column density derived via joint fitting of several sources may not always be a good estimate of their "typical" column density. The presence of even one source with significant low-energy flux can bias the joint fitting column density downward, since such flux is highly inconsistent with a strong photoelectric absorption cutoff at low energy.
}

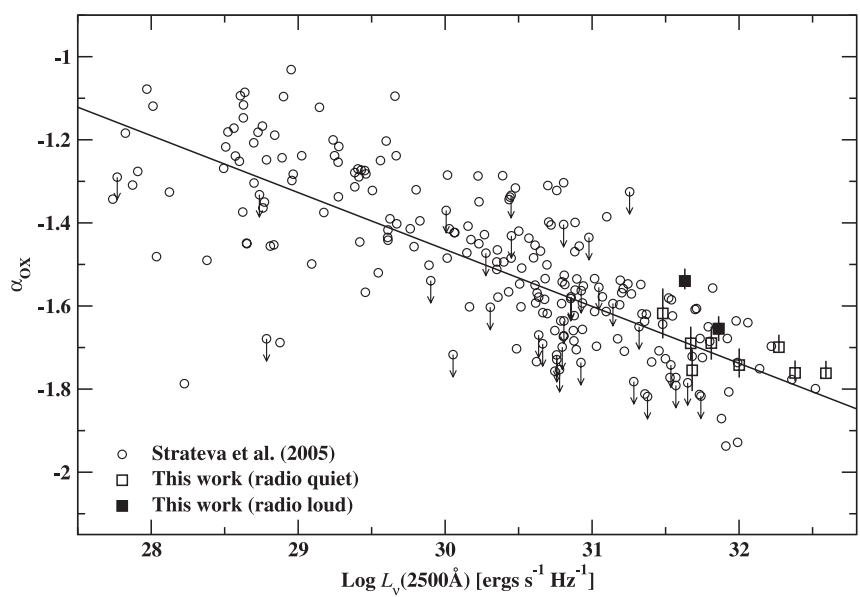

FIG. 4.-Optical-to-X-ray SED parameter, $\alpha_{\text {ox }}$, vs. luminosity density at $2500 \AA$. The 10 AGNs studied in this work (squares) follow the Strateva et al. (2005) $\alpha_{\mathrm{ox}}-L_{\nu}(2500 \AA)$ relation (solid line $), \alpha_{\mathrm{ox}}=-0.136 L_{\nu}(2500 \AA)+2.616$. The two moderately radio-loud sources presented in this work, PSS $0121+0347$ and SDSS 0210-0018, are the only such sources on this diagram and are marked with filled squares. The reason why most of our sources lie above the best-fit line may be attributed to the fact that we specifically targeted X-ray-bright sources.

reflection hump (see Table 4). These are the best constraints to date on these properties of $z>4$ AGNs. Due to the high mean X-ray luminosity $\left[\left\langle\log L_{2-10 \mathrm{keV}}\left(\mathrm{ergs} \mathrm{s}^{-1}\right)\right\rangle \simeq 45.5\right]$ of our sample, both the $\mathrm{Fe} \mathrm{K} \alpha$ line and Compton hump are expected to be relatively weak. Using the recent Page et al. (2004a) anticorrelation between $\mathrm{EW}(\mathrm{Fe} \mathrm{K} \alpha)$ and $L_{2-10 \mathrm{keV}}$ (the so-called Fe $\mathrm{K} \alpha$ Baldwin relation), we find an empirically expected range of $\mathrm{EW}(\mathrm{Fe} \mathrm{K} \alpha) \sim 25-80 \mathrm{eV}$ for our sources, several times lower than our upper limit (but see also Jimenez-Bailón et al. 2005). Given our results, the $\mathrm{EW}(\mathrm{Fe} \mathrm{K} \alpha) \approx 1 \mathrm{keV}$ for SDSS $1306+0356$ (see $\S 3$ ) appears remarkable and should be investigated with better data. The constraints that we obtain on the Compton reflection component, $R \lesssim 1.2$, are consistent with those obtained for some $z \simeq 0-1$ AGNs (e.g., Page et al. 2004b) but are significantly lower than the value $R=2.87 \pm 0.96$ found in PG $1247+267$ at $z=2.04$, the most luminous source in which such a component was significantly detected (Page et al. 2004b).

\subsection{Optical-to-X-Ray SED}

For each of the 10 AGNs we computed the optical-to-X-ray SED parameter, $\alpha_{\mathrm{ox}}$, defined as

$$
\alpha_{\mathrm{ox}}=\frac{\log \left(f_{2 \mathrm{keV}} / f_{2500 \AA}\right)}{\log \left(\nu_{2 \mathrm{keV}} / \nu_{2500 \AA}\right)},
$$

where $f_{2 \mathrm{keV}}$ and $f_{2500 \AA}$ are the flux densities at rest-frame $2 \mathrm{keV}$ and $2500 \AA$, respectively (e.g., Tananbaum et al. 1979).

The $\alpha_{\text {ox }}$ values and their $1 \sigma$ errors appear in Table 3. The errors on $\alpha_{\text {ox }}$ were computed following the "numerical method" described in $\S 1.7 .3$ of Lyons (1991), taking into account the uncertainties on the X-ray power-law normalizations and photon indices, and the effects of possible changes in the UV-optical slope (from $\alpha=-0.5$ to -0.79 ). In Figure 4 we plot $\alpha_{\text {ox }}$ for our sources against their optical luminosities and include a sample of 228 radio-quiet AGNs (mainly from the SDSS) from Strateva et al. (2005) in the diagram. Also plotted in Figure 4 is the bestfit line of the Strateva et al. (2005) $\alpha_{\mathrm{ox}}-L_{\nu}(2500 \AA)$ relation (eq. [6] of Strateva et al. 2005). The $\alpha_{\text {ox }}$ values of the 10 AGNs presented in this work, which represent extreme luminosities and redshifts, generally follow that relation, although most of 
TABLE 6

Two-Epoch X-Ray Fluxes Obtained by XMM-Newton and Chandra

\begin{tabular}{|c|c|c|c|c|c|c|}
\hline \multirow[b]{2}{*}{ AGN } & \multicolumn{2}{|c|}{ FIRST ЕPOCH } & \multicolumn{2}{|c|}{ SECOND Epoch } & \multirow[b]{2}{*}{$\begin{array}{c}\Delta t^{\mathrm{b}} \\
\text { (days) }\end{array}$} & \multirow[b]{2}{*}{$\chi^{2 \mathrm{c}}$} \\
\hline & 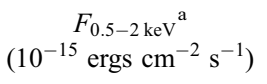 & Reference & 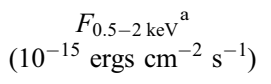 & Reference & & \\
\hline PSS $0121+0347 \ldots \ldots \ldots \ldots \ldots \ldots \ldots$ & $34.7_{-4.4}^{+5.4}$ & 1 & $17.02_{-2.28}^{+2.19}$ & 2 & 135 & 20.40 \\
\hline SDSS $0210-0018 \ldots \ldots \ldots \ldots \ldots \ldots$ & $28.1_{-6.0}^{+8.4}$ & 3 & $14.37_{-1.76}^{+1.70}$ & 2 & 258 & 16.35 \\
\hline SDSS $0231-0728 \ldots \ldots \ldots \ldots \ldots \ldots$ & $21.3_{-4.6}^{+5.0}$ & 4 & $4.68_{-1.29}^{+1.35}$ & 2 & 73 & 41.42 \\
\hline PSS $0926+3055 \ldots \ldots \ldots \ldots \ldots \ldots \ldots$ & $27.7_{-4.1}^{+4.5}$ & 1 & $39.04_{-2.54}^{+2.57}$ & 2 & 186 & 6.43 \\
\hline PSS $1326+0743 \ldots \ldots \ldots \ldots \ldots \ldots \ldots$ & $24.4_{-3.6}^{+4.1}$ & 1 & $27.89_{-2.52}^{+1.94}$ & 2 & 139 & 0.64 \\
\hline SDSS $1030+0524 \ldots \ldots \ldots \ldots \ldots \ldots$ & $2.43_{-0.99}^{+1.48}$ & 5 & $3.09_{-0.52}^{+0.53}$ & 6 & 68 & 0.45 \\
\hline SDSS $1306+0356 \ldots \ldots \ldots \ldots \ldots \ldots$ & $4.63_{-1.33}^{+1.77}$ & 5 & $2.74_{-0.53}^{+0.56}$ & 7 & 96 & 3.35 \\
\hline
\end{tabular}

${ }^{\text {a }}$ Galactic absorption-corrected flux in the observed $0.5-2 \mathrm{keV}$ band. Errors represent $90 \%$ confidence limits on the fluxes.

${ }^{\mathrm{b}}$ Rest-frame time difference between the two epochs.

${ }^{c}$ Variability statistic; see $\S 5.5$.

References.- (1) Vignali et al. 2003a; (2) this work; (3) Vignali et al. 2001; (4) Vignali et al. 2003b; (5) Brandt et al. 2002; (6) Farrah et al. 2004; (7) Schwartz \& Virani 2004.

them lie above the best-fit line. This may be due to a selection bias, since we have specifically targeted X-ray-bright sources.

\subsection{X-Ray Variability}

Using the event files of our XMM-Newton observations, we searched for rapid ( $\sim 1 \mathrm{hr}$ timescale in the rest frame) variability of the AGNs by applying Kolmogorov-Smirnov tests to the lists of photon arrival times. No significant variations were detected, and the fractional variability of the count rate light curves was smaller than $15 \%$ in all cases. To test whether the X-ray flux exhibits long-term (months to years) variations in our sample, we list in Table 6 pairs of two-epoch $0.5-2 \mathrm{keV}$ fluxes for seven $z>4$ sources that were observed with Chandra in the first epoch and with either XMM-Newton or Chandra in the second epoch. The relative uncertainties between flux measurements obtained with XMM-Newton versus those obtained with Chandra are considered to be the smallest among different X-ray observatories and are normally $\lesssim 10 \%$ (Snowden 2002; S. Snowden 2005 , private communication). Table 6 also lists the rest-frame temporal separation between the two observations, and a $\chi^{2}$ statistic to quantify the significance of variation between the two epochs. The null hypothesis is that the flux in each epoch is equal to the mean flux of the two epochs. A larger $\chi^{2}$ implies higher significance of variability, and values larger than 2.71 (6.63) imply that the two fluxes are inconsistent within their uncertainties; i.e., there is significant variability at a $90 \%(99 \%)$

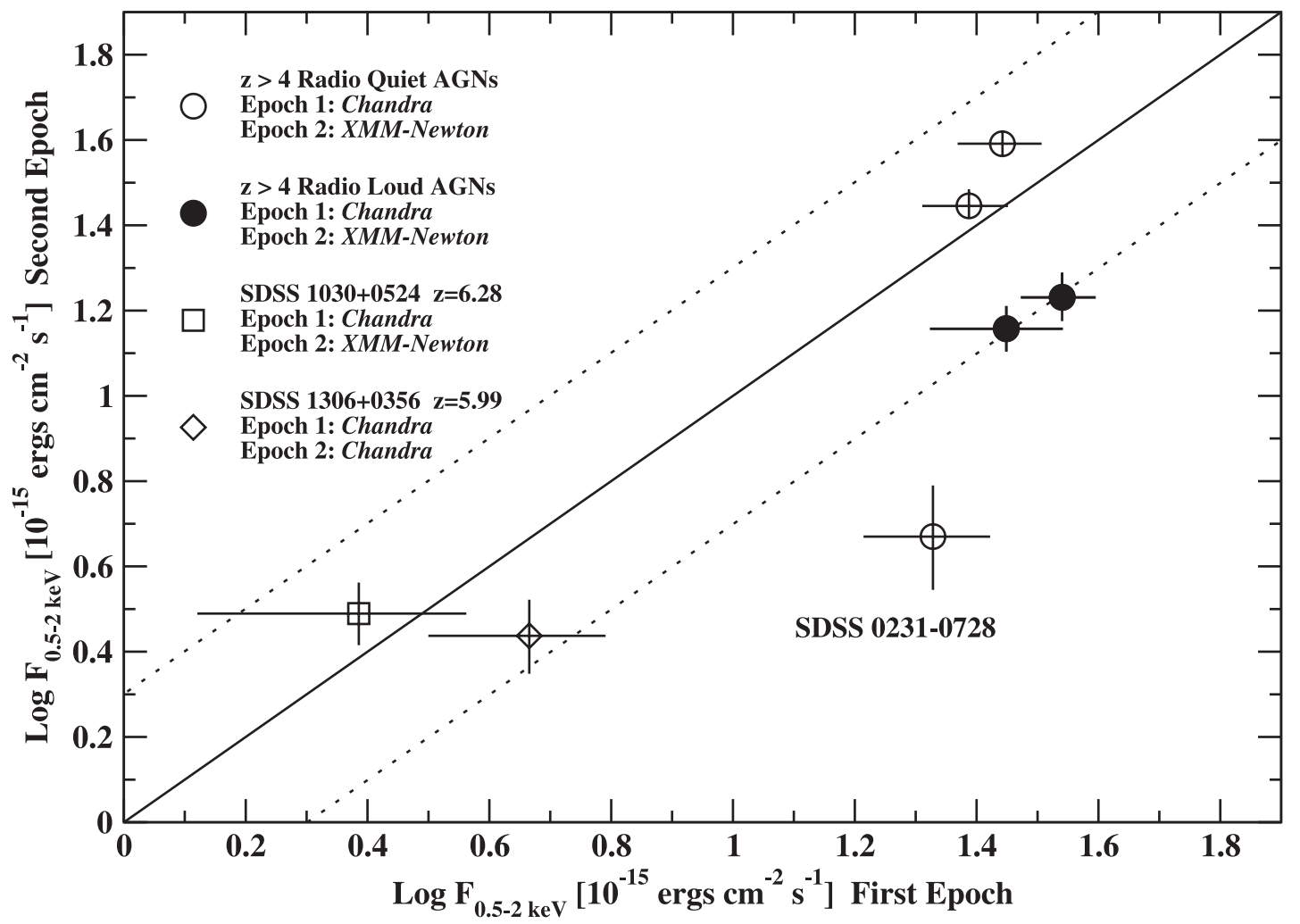

FIG. 5.-Two-epoch Galactic absorption-corrected $0.5-2 \mathrm{keV}$ fluxes for seven of the $z>4$ AGNs in our sample. The solid line marks the $1: 1$ flux ratio, and the two

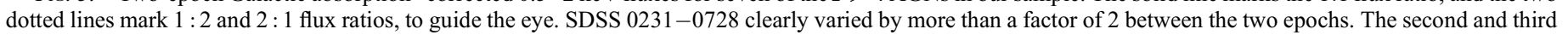
most variable sources, PSS 0121+0347 and SDSS 0210-0018, are moderately radio-loud and are marked with filled circles. 
confidence level (e.g., Avni 1976). Of the seven AGNs studied, only two sources, PSS $1326+0743$ and SDSS $1030+0524$, do not show a significant (i.e., at $\geq 90 \%$ confidence) long-term variation. Figure 5 plots the fluxes of the second epoch against those of the first, as given in Table 6 .

While most AGNs varied by no more than a factor of $\approx 2$ between the two epochs, one source, SDSS 0231-0728, faded by a factor of $\sim 4$ between the first (Chandra) observation and the second (XMM-Newton) one. This flux change occurred over a rest-frame period of 73 days. This is the largest change in $\mathrm{X}$-ray flux observed in a $z>4$ radio-quiet AGN (e.g., Paolillo et al. 2004). Given the optical flux of the source, and using the Vignali et al. (2003b) relation between optical and X-ray fluxes, it is likely that this source was caught in an X-ray high state in the first epoch (Vignali et al. 2003b), since its X-ray flux in the second epoch (this work) agrees with the value predicted from its optical flux (assuming that the optical flux is nearly constant). Vignali et al. (2003b) also noted that SDSS 0231-0728 was $\mathrm{X}$-ray brighter than expected (see their Fig. 5). The spectral slope of the source also shows a possible indication of flattening from $\Gamma=2.8_{-0.95}^{+1.10}$ to $1.85_{-0.31}^{+0.33}$ between the two epochs, but the significance is only $\sim 1 \sigma$ due to the limited number of counts $(\sim 25)$ in the first Chandra snapshot observation. This is a tentative indication for a transition from a soft/high state to a hard/low state in this source, as has been seen for a few local AGNs (e.g., Guainazzi et al. 1998; Maccarone et al. 2003).

\section{SUMMARY AND FUTURE PROSPECTS}

Moderate- to high-quality X-ray spectra of 10 luminous AGNs at $z>4$, including five new $X M M$-Newton observations, have been analyzed to extract X-ray spectral properties and search for flux variations. We found that the X-ray power-law photon index does not depend on either luminosity or redshift, broadly consistent with the lack of multiwavelength AGN SED evolution, and it has no detectable intrinsic dispersion at $z>4$. Upper limits placed on the intrinsic absorption in eight radioquiet $z>4$ AGNs show that, on average, the X-ray light of luminous radio-quiet AGNs at $z>4$ is not more absorbed than in nearby AGNs. All this suggests that the X-ray production mechanism and the central environment in radio-quiet AGNs do not depend on luminosity and have not evolved over cosmic time. We also place constraints on the strength of a putative narrow $\mathrm{Fe} \mathrm{K} \alpha$ line and constrain the relative strength of the
Compton reflection hump at high rest-frame X-ray energies. Tighter constraints on those X-ray properties of luminous AGNs at $z>4$, with existing X-ray observatories, can only be achieved by lengthy (several $100 \mathrm{ks}$ ) observations of the most luminous sources.

A search for short-timescale $(\sim 1 \mathrm{hr}) \mathrm{X}$-ray variability found no significant variations, but on longer timescales (months to years) we have found significant variations in five of our sources, noting one extreme radio-quiet source in which the X-ray flux dropped by a factor of $\sim 4$ over a 73 day rest-frame period; this is the most extreme $\mathrm{X}$-ray variation ever observed for a radioquiet AGN at $z>4$. X-ray monitoring of SDSS 0231-0728 and other AGNs at high redshift will enable us to link the variability properties of high-redshift AGNs with some of their fundamental properties, such as BH mass, luminosity, and accretion rate, as is done at lower redshift (e.g., O'Neill et al. 2005). Two recent studies have reported that the X-ray flux variations of high-redshift AGNs are larger than those of their nearby counterparts (Manners et al. 2002; Paolillo et al. 2004). The data presented in this paper do not allow us to test this claim. Multiple-epoch X-ray observations of a large sample of high-redshift radio-quiet AGNs are required to investigate their variability properties and compare them with those of the local AGN population.

This work is based on observations obtained with $X M M$ Newton, an ESA science mission with instruments and contributions directly funded by ESA member states and the US (NASA). We are grateful to Bret Lehmer and Divas Sanwal for help with the $X M M-N e w t o n$ data reduction. We also thank Aaron Steffen, Iskra Strateva, and Dan Vanden Berk for useful discussions. An anonymous referee is gratefully acknowledged for helping to improve the presentation of this paper. We gratefully acknowledge the financial support of NASA grant NNG04GF57G (O. S., W. N. B., D. P. S.), NASA LTSA grant NAG5-13035 (W. N. B., D. P. S.), and NSF grants AST 03-07582 (D. P. S.), AST 03-07409 (M. A. S.), and AST 03-07384 (X. F.). X. F. acknowledges support from an Alfred P. Sloan Research Fellowship and a David and Lucile Packard Fellowship in Science and Engineering. C. V. acknowledges support from MIUR cofin grant 03-02023 and INAF/PRIN grant 270/2003.
Akritas, M. G., \& Bershady, M. A. 1996, ApJ, 470, 706

Anders, E., \& Grevesse, N. 1989, Geochim. Cosmochim. Acta, 53, 197

Anderson, S. F., et al. 2001, AJ, 122, 503

Arnaud, K. A. 1996, in ASP Conf. Ser. 101, Astronomical Data Analysis Software and Systems V, ed. G. H. Jacoby \& J. Barnes (San Francisco: ASP), 17

Avni, Y. 1976, ApJ, 210, 642

Balucinska-Church, M., \& McCammon, D. 1992, ApJ, 400, 699

Barger, A. J., Cowie, L. L., Mushotzky, R. F., Yang, Y., Wang, W.-H., Steffen, A. T., \& Capak, P. 2005, AJ, 129, 578

Bechtold, J., et al. 2003, ApJ, 588, 119

Bertoldi, F., Carilli, C. L., Cox, P., Fan, X., Strauss, M. A., Beelen, A., Omont, A., \& Zylka, R. 2003, A\&A, 406, L55

Brandt, W. N., \& Hasinger, G. 2005, ARA\&A, in press (astro-ph/0501058)

Brandt, W. N., Vignali, C., Lehmer, B. D., Lopez, L. A., Schneider, D. P., \& Strateva, I. V. 2005, in Growing Black Holes, ed. A. Merloni, S. Nayakshin, $\&$ R. Sunyaev (Berlin: Springer), in press (astro-ph/0411355)

Brandt, W. N., et al. 2002, ApJ, 569, L5 2004, Adv. Space Res., 34, 2478

Cappi, M., Matsuoka, M., Comastri, A., Brinkmann, W., Elvis, M., Palumbo, G. G. C., \& Vignali, C. 1997, ApJ, 478, 492

Carilli, C. L., et al. 2001, ApJ, 555, 625

\section{REFERENCES}

Croom, S. M., Smith, R. J., Boyle, B. J., Shanks, T., Miller, L., Outram, P. J., \& Loaring, N. S. 2004, MNRAS, 349, 1397

Dickey, J. M., \& Lockman, F. J. 1990, ARA\&A, 28, 215

Dietrich, M., Hamann, F., Shields, J. C., Constantin, A., Heidt, J., Jäger, K., Vestergaard, M., \& Wagner, S. J. 2003, ApJ, 589, 722

Djorgovski, S. G., Gal, R. R., Odewahn, S. C., de Carvalho, R. R., Brunner, R., Longo, G., \& Scaramella, R. 1998, in Wide Field Surveys in Cosmology, 14th IAP Meeting, ed. S. Colombi, Y. Mellier, \& B. Raban (Gif-sur-Yvette: Editions Frontières), 89

Djorgovski, S. G., Stern, D., Mahabal, A. A., \& Brunner, R. 2003, ApJ, 596, 67 Fabian, A. C., Celotti, A., Pooley, G., Iwasawa, K., Brandt, W. N., McMahon, R. G., \& Hoenig, M. D. 1999, MNRAS, 308, L6

Fan, X., Narayanan, V. K., Strauss, M. A., White, R. L., Becker, R. H., Pentericci, L., \& Rix, H. 2002, AJ, 123, 1247

Fan, X., et al. 1999, AJ, 118, 1 2001, AJ, 121, 31 2003, AJ, 125, 1649

Farrah, D., Priddey, R., Wilman, R., Haehnelt, M., \& McMahon, R. 2004, ApJ, 611, L13

Ferrero, E., \& Brinkmann, W. 2003, A\&A, 402, 465

Grupe, D., Mathur, S., Wilkes, B., \& Elvis, M. 2004, AJ, 127, 1 
Grupe, D., Mathur, S., Wilkes, B., \& Osmer, P. 2005, AJ, submitted (astro-ph/ 0501521)

Guainazzi, M., et al. 1998, A\&A, 339, 327

Hennawi, J., et al. 2005, AJ, submitted (astro-ph/0504535)

Ivezić, Ž., et al. 2002, AJ, 124, 2364

Jansen, F., et al. 2001, A\&A, 365, L1

Jimenez-Bailón, E., Piconcelli, E., Guainazzi, M., Schartel, N., RodríguezPascual, P. M., \& Santos-Lleó, M. 2005, A\&A, 435, 449

Kaspi, S., Brandt, W. N., \& Schneider, D. P. 2000, AJ, 119, 2031

Kellermann, K. I., Sramek, R., Schmidt, M., Shaffer, D. B., \& Green, R. 1989, AJ, 98, 1195

Kirsch, M. G. F., et al. 2004, Proc. SPIE, 5488, 103

Laor, A., Fiore, F., Elvis, M., Wilkes, B. J., \& McDowell, J. C. 1997, ApJ, 477, 93

Lyons, L. 1991, Data Analysis for Physical Science Students (Cambridge: Cambridge Univ. Press)

Maccacaro, T., Gioia, I. M., Wolter, A., Zamorani, G., \& Stocke, J. T. 1988, ApJ, 326, 680

Maccarone, T. J., Gallo, E., \& Fender, R. 2003, MNRAS, 345, L19

Magdziarz, P., \& Zdziarski, A. A. 1995, MNRAS, 273, 837

Maiolino, R., Oliva, E., Ghinassi, F., Pedani, M., Mannucci, F., Mujica, R., \& Juarez, Y. 2004, A\&A, 420, 889

Manners, J., Almaini, O., \& Lawrence, A. 2002, MNRAS, 330, 390

O’Neill, P. M., Nandra, K., Papadakis, I. E., \& Turner, T. J. 2005, MNRAS, 358, 1405

Page, K. L., O’Brien, P. T., Reeves, J. N., \& Turner, M. J. L. 2004a, MNRAS, 347,316

Page, K. L., Reeves, J. N., O’Brien, P. T., Turner, M. J. L., \& Worrall, D. M. 2004b, MNRAS, 353, 133

Paolillo, M., Schreier, E. J., Giacconi, R., Koekemoer, A. M., \& Grogin, N. A. 2004, ApJ, 611, 93

Pentericci, L., et al. 2003, A\&A, 410, 75

Petric, A. O., Carilli, C. L., Bertoldi, F., Fan, X., Cox, P., Strauss, M. A., Omont, A., \& Schneider, D. P. 2003, AJ, 126, 15

Piconcelli, E., Jimenez-Bailón, E., Guainazzi, M., Schartel, N., RodríguezPascual, P. M., \& Santos-Lleó, M. 2005, A\&A, 432, 15
Porquet, D., Reeves, J. N., O’Brien, P., \& Brinkmann, W. 2004, A\&A, 422, 85 Reynolds, C. S., \& Nowak, M. A. 2003, Phys. Rep., 377, 389

Richards, G. T., et al. 2002, AJ, 123, 2945

Schneider, D. P., Schmidt, M., \& Gunn, J. E. 1989, AJ, 98, 1507

Schneider, D. P., et al. 2005, AJ, 130, 367

Schwartz, D. A., \& Virani, S. N. 2004, ApJ, 615, L21

Snowden, S. L. 2002, in ESA WPP Conf. Ser. CD-ROM, New Visions of the $\mathrm{X}$-Ray Universe in the XMM-Newton and Chandra Era (Noordwijk: ESTEC) Spergel, D. N., et al. 2003, ApJS, 148, 175

Steffen, A. T., Barger, A. J., Cowie, L. L., Mushotzky, R. F., \& Yang, Y. 2003, ApJ, 596, L23

Stern, D., Djorgovski, S. G., Perley, R. A., de Carvalho, R. R., \& Wall, J. V. 2000, AJ, 119, 1526

Storrie-Lombardi, L. J., McMahon, R. G., Irwin, M. J., \& Hazard, C. 1996, ApJ, 468, 121

Strateva, I., Brandt, W. N., Schneider, D. P., Vanden Berk, D., \& Vignali, C. 2005, AJ, 130, 387

Tananbaum, H., et al. 1979, ApJ, 234, L9

Vanden Berk, D., Yip, C., Connolly, A., Jester, S., \& Stoughton, C. 2004, in ASP Conf. Ser. 311, AGN Physics with the Sloan Digital Sky Survey, ed. G. T. Richards \& P. B. Hall (San Francisco: ASP), 21

Vignali, C., Bauer, F. E., Alexander, D. M., Brandt, W. N., Hornschemeier, A. E., Schneider, D. P., \& Garmire, G. P. 2002, ApJ, 580, L105

Vignali, C., Brandt, W. N., Fan, X., Gunn, J. E., Kaspi, S., Schneider, D. P., \& Strauss, M. A. 2001, AJ, 122, 2143

Vignali, C., Brandt, W. N., Schneider, D. P., Garmire, G. P., \& Kaspi, S. 2003a, AJ, 125, 418

Vignali, C., Brandt, W. N., Schneider, D. P., \& Kaspi, S. 2005, AJ, 129, 2519

Vignali, C., et al. 2003b, AJ, 125, 2876

Walter, F., et al. 2003, Nature, 424, 406

Wilkes, B. J., \& Elvis, M. 1987, ApJ, 323, 243

Worsley, M. A., Fabian, A. C., Celotti, A., \& Iwasawa, K. 2004a, MNRAS, 350, L67

Worsley, M. A., Fabian, A. C., Turner, A. K., Celotti, A., \& Iwasawa, K. 2004b, MNRAS, 350, 207

York, D. G., et al. 2000, AJ, 120, 1579 